\title{
Front Matter: Volume 10596
}

, "Front Matter: Volume 10596," Proc. SPIE 10596, Behavior and Mechanics of Multifunctional Materials and Composites XII, 1059601 (3 May 2018); doi: $10.1117 / 12.2324810$

SPIE Event: SPIE Smart Structures and Materials + Nondestructive Evaluation and Health Monitoring, 2018, Denver, Colorado, United States 


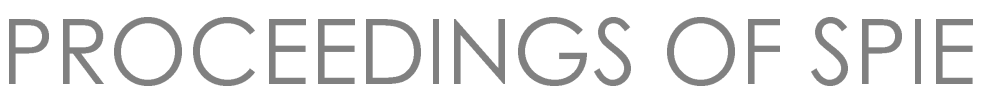

\section{Behavior and Mechanics of Multifunctional Materials and Composites XII}

Hani E. Naguib

Editors

5-8 March 2018

Denver, Colorado, United States

Sponsored by

SPIE

Co-sponsored by

OZ Optics, Ltd. (United States)

Polytec, Inc. (United States)

Cooperating Organizations

Jet Propulsion Laboratory (United States)

Colorado Photonics Industry Association (United States)

Published by

SPIE

Volume 10596 
The papers in this volume were part of the technical conference cited on the cover and title page. Papers were selected and subject to review by the editors and conference program committee. Some conference presentations may not be available for publication. Additional papers and presentation recordings may be available online in the SPIE Digital Library at SPIEDigitallibrary.org.

The papers reflect the work and thoughts of the authors and are published herein as submitted. The publisher is not responsible for the validity of the information or for any outcomes resulting from reliance thereon.

Please use the following format to cite material from these proceedings:

Author(s), "Title of Paper," in Behavior and Mechanics of Multifunctional Materials and Composites XII, edited by Hani E. Naguib, Proceedings of SPIE Vol. 10596 (SPIE, Bellingham, WA, 2018) Seven-digit Article CID Number.

ISSN: 0277-786X

ISSN: 1996-756X (electronic)

ISBN: 9781510616882

ISBN: 9781510616899 (electronic)

Published by

SPIE

P.O. Box 10, Bellingham, Washington 98227-0010 USA

Telephone +1360 676 3290 (Pacific Time) · Fax +1 3606471445

SPIE.org

Copyright @ 2018, Society of Photo-Optical Instrumentation Engineers.

Copying of material in this book for internal or personal use, or for the internal or personal use of specific clients, beyond the fair use provisions granted by the U.S. Copyright Law is authorized by SPIE subject to payment of copying fees. The Transactional Reporting Service base fee for this volume is $\$ 18.00$ per article (or portion thereof), which should be paid directly to the Copyright Clearance Center (CCC), 222 Rosewood Drive, Danvers, MA 01923. Payment may also be made electronically through CCC Online at copyright.com. Other copying for republication, resale, advertising or promotion, or any form of systematic or multiple reproduction of any material in this book is prohibited except with permission in writing from the publisher. The CCC fee code is 0277 $786 \mathrm{X} / 18 / \$ 18.00$.

Printed in the United States of America.

Publication of record for individual papers is online in the SPIE Digital Library.

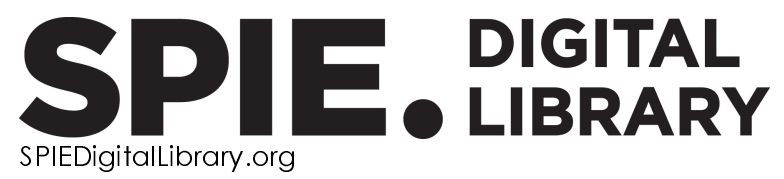

Paper Numbering: Proceedings of SPIE follow an e-First publication model. A unique citation identifier (CID) number is assigned to each article at the time of publication. Utilization of CIDs allows articles to be fully citable as soon as they are published online, and connects the same identifier to all online and print versions of the publication. SPIE uses a seven-digit CID article numbering system structured as follows:

- The first five digits correspond to the SPIE volume number.

- The last two digits indicate publication order within the volume using a Base 36 numbering system employing both numerals and letters. These two-number sets start with $00,01,02,03,04$, 05, 06, 07, 08, 09, OA, OB ... 0Z, followed by 10-1Z, 20-2Z, etc. The CID Number appears on each page of the manuscript. 


\title{
Contents
}

\author{
vii Authors \\ ix Conference Committee
}

\section{TUESDAY PLENARY SESSION}

1059603 Current and future needs and research for composite materials NDE (Plenary Paper) [10596-500]

\section{SESSION 1 AUXETIC AND METAMATERIALS}

1059604 Fractional viscoelasticity of soft elastomers and auxetic foams [10596-1]

1059606 Boundary modes in quasiperiodic elastic structures [10596-4]

\section{SESSION 2 SHAPE MEMORY MATERIALS I}

1059608 Damage evolution during actuation fatigue in shape memory alloys (SPIE Best Student Paper Award) [10596-5]

1059609 Shape memory alloy-actuated bistable composites for morphing structures [10596-7]

10596 OA Analytical model for a laminated shape memory alloy beam with piezoelectric layers [10596-9]

\section{SESSION 3 ADDITIVE MANUFACTURING I}

$10596 \mathrm{OH}$ Influence of SLM on compressive response of NiTi scaffolds [10596-13]

$10596 \mathrm{OJ}$ Electrical conductivity and piezoresistive response of 3D printed thermoplastic polyurethane/multiwalled carbon nanotube composites [10596-15]

\section{SESSION 4 ORIGAMI MATERIALS}

1059600 Buckling behavior of origami unit cell facets under compressive loads [10596-19]

\section{SESSION 5 DIELECTRICS AND PIEZOLECTRICS}

10596 OR Phenomenological model for coupled multi-axial piezoelectricity [10596-23] 
10596 OS Uncertainty quantification for PZT bimorph actuators [10596-24]

10596 OT Active subspace uncertainty quantification for a polydomain ferroelectric phase-field model [10596-25]

\section{SESSION 6 ADDITIVE MANUFACTURING II}

10596 OV Solvent sensitivity of smart 3D-printed nanocomposite liquid sensors [10596-26]

10596 OW Selective laser melting of Ni-rich NiTi: selection of process parameters and the superelastic response [10596-27]

10596 OX Ultrasonic alignment of microparticles in nozzle-like geometries [10596-28]

$10596 \mathrm{OZ}$ Electromechanical modeling and experimental verification of a directly printed nanocomposite [10596-30]

1059610 Mechanical evaluation of the SLM fabricated, stiffness-matched, mandibular bone fixation plates [10596-31]

1059611 Materials challenges for repeatable RF wireless device reconfiguration with microfluidic channels [10596-32]

\section{SESSION 7 SHAPE MEMORY MATERIALS II}

1059612 Experimental and numerical investigation of the stable crack growth regime under pseudoelastic loading in shape memory alloys [10596-33]

1059613 Modeling of NitiHf using finite difference method [10596-34]

1059616 Corrosion monitoring of NiTi alloy with small-amplitude potential intermodulation technique [10596-37]

\section{SESSION 8 NANOCOMPOSITES APPLICATIONS}

1059618 Development and characterization of reduced graphene oxide films for transient electronics [10596-40]

10596 1A Anisotropic thermal conductivity of aligned GNPs/polymer composites: a finite element approach [10596-41]

\section{SESSION 9 MAGNETOSTRICTIVE MATERIALS}

10596 1C Analysis of the mechanical behavior of single wall carbon nanotubes by a modified molecular structural mechanics model incorporating an advanced chemical force field [10596-43]

iv 
10596 ID Uncertainty-enabled design of electromagnetic reflectors with integrated shape control [10596-44]

10596 1E Inverse magnetostrictive characteristics of Fe-Co composite materials using gas-nitriding process [10596-45]

$105961 G$ Performance prediction for a magnetostrictive actuator using a simplified model [10596-47]

$10596 \mathrm{1H}$ "Shape function + memory mechanism"-based hysteresis modeling of magnetorheological fluid actuators [10596-48]

\section{SESSION 10 MECHANICS OF MULTIFUNCTIONAL MATERIALS}

$1059611 \quad$ Synchronous separation, seaming, sealing, and sterilization (S4) using brazing for sample containerization and planetary protection [10596-49]

$105961 \mathrm{~J}$ The effects of surface topography control using liquid crystal elastomers on bodies in flow [10596-50]

$105961 \mathrm{~K}$ Strength and failure analysis of composite-to-composite adhesive bonds with different surface treatments [10596-51]

$10596 \mathrm{lL}$ Isolation of aramid nanofibers for strong nanocomposites and multiscale fiber reinforcements [10596-52]

$105961 \mathrm{M}$ Influence of material anisotropy on the hydroelastic response of composite plates in water [10596-53]

$105961 \mathrm{~N}$ Evaluation of thermally aged carbon fiber/epoxy composites using acoustic emission, electrical resistance, contact angle, and thermogram [10596-54]

\section{SESSION $11 \quad$ PHYSICALLY RESPONSIVE MATERIALS}

1059610 Various design approaches to achieve electric field-driven segmented folding actuation of electroactive polymer (EAP) sheets [10596-71]

10596 IP Time-dependent chemo-electro-mechanical behavior of hydrogel-based structures [10596-55]

\section{SESSION 12 MULTIFUNCTIONAL MATERIALS APPLICATIONS}

10596 is Optimal power, power limit, and damping of piezoelectric vibration energy harvesters [10596-72]

10596 IT Interfacial characterization of flexible hybrid electronics [10596-73] 
$105961 \mathrm{U}$ Evaluation of oil-leakage of multi-layered resin-hose clamped with metal nipple and sleeve [10596-59]

10596 IW Effect of the carbonyl iron particles on acoustic absorption properties of magnetic polyurethane foam [10596-61]

10596 1X The electrical resistivity and percolation threshold of MWCNTs/polymer composites filled with a few aligned carbonyl iron particles [10596-62]

10596 1Y Numerical model for an epoxy beam reinforced with superelastic shape memory alloy wires [10596-63]

$10596 \mathrm{lZ}$ Characteristics of energy harvesting using $\mathrm{BaTiO}_{3} / \mathrm{Cu}$ laminates with changes in temperature [10596-64]

1059622 Modeling the effects of strain profiles and defects on precessional magnetic switching in multiferroic heterostructures [10596-67]

1059623 Safety concerns in composite manufacturing and machining [10596-68]

1059624 Sustainability of fiber reinforced laminate and honeycomb composites in manufacturing industries [10596-69] 


\section{Authors}

Numbers in the index correspond to the last two digits of the seven-digit citation identifier (CID) article numbering system used in Proceedings of SPIE. The first five digits reflect the volume number. Base 36 numbering is employed for the last two digits and indicates the order of articles within the volume. Numbers start with 00, 01, 02, 03, 04, 05, 06, 07, 08, 09, OA, OB...0Z, followed by 10-1Z, 20-2Z, etc.

Ahmed, Saad, 10

Akcabay, Deniz Tolga, $1 \mathrm{M}$

Alamir, Mohammed, 1K, 23, 24

Aliheidari, Nahal, OV

Alonayni, Abdullah, 1K, 23, 24

Ameli, Amir, OJ, OV

Amerinatanzi, Amirhesam, OH, OW, 10

Amirkhizi, Alireza $\vee ., 1 T$

Arruda, José R. F., 06

Asmatulu, Eylem, 1K, 23, 24

Asmatulu, Ramazan, 1K

Badescu, Mircea, 11

Baek, Y. M., $1 \mathrm{~N}$

Bai, Xian-XU, $1 \mathrm{H}$

Bao, Xiaoqi, 11

Bar-Cohen, Yoseph, 11

Baxevanis, Theocharis, 12

Beblo, Richard, 1J

Bhatkar, Omkar, 18

Bravo, Nikolas, OS

Cai, Fei-Long, $1 \mathrm{H}$

Carman, Gregory P., 22

Castaneda, H., 16

Chavez, Andres C., 22

Chen, Peng, $1 \mathrm{H}$

Chillara, Venkata Siva C., 09

Chung, Jooyeon, 1D

Cramer, K. Elliott, 03

Crews, John, OS

Dapino, Marcelo J., 09

Dauson, Erin R., OX

Dean, David, 10

DeVries, K. L., $1 \mathrm{~N}$

Dong, Shuai, $1 \mathrm{X}$

Eberhardt, Oliver, 1C

Elahinia, Mohammad, $\mathrm{OH}, \mathrm{OW}, 10,13$

Farjam, Nazanin, 13

Frecker, Mary, 10

Fujii, Toru, $1 \mathrm{U}$

Fujishita, Yushi, $1 \mathrm{U}$

Geng, Jialu, 1A, 1W

Griffin, Anthony S., 11

Guin, Tyler, 1J

Haghgouyan, Behrouz, 12

Haque, Samiul, 1D

Hayrettin, Ceylan, 12

Heard, Robert A., OX

Hohimer, Cameron J., OJ

Hong, Jonathan, 10
Jahadakbar, Ahmadreza, OH, OW, 10

Jape, Sameer, 12

Jones, Nicholas J., $1 \mathrm{G}$

Karaca, Haluk, OH, OW, 13

Karaman, Ibrahim, 12

Kim, Daewa, 1D

Kim, J. H., 1N

Kindrat, Laszlo P., 1D

Kopatz, Jessica, 10

Kshad, Mohamed Ali Emhmed, 00

Kuian, Mykhailo, 1D

Kundu, Auni A., 22

Kusu, Fuko, $1 \mathrm{U}$

Kutzer, Thomas, 11

Lagoudas, Dimitris C., 08, 12, 16

Leichsenring, Peter, $1 \mathrm{P}$

Leon, Lider S., OT

Li, Bin, $1 \mathrm{~A}$

Li, Rui, 1 A

Liao, Yabin, $1 \mathrm{~s}$

Lin, Jiajun, $1 \mathrm{~L}$

Lindsey, Cameron, 11

Liu, Sijing, 1D

Lynch, Christopher S., 22

Malakooti, Mohammad H., $1 \mathrm{~L}$

Massad, Jordan E., 1D

Matsuoka, Kenta, $1 \mathrm{U}$

Matsushita, Masato, $1 \mathrm{U}$

Mehrabi, Reza, 13

Mikheev, Vikenty, 1D

Miles, Paul, 04, OT

Mirzaeifar, Reza, 13

Mo, Changki, OJ

Mohajeri, M., 16

Mori, K., $1 Z$

Nafari, Alireza, $\mathrm{OZ}$

Naguib, Hani E., $0 \mathrm{O}$

Najafian, Sara, $1 T$

Nakajima, Kenya, $1 \mathrm{E}$

Nakamura, Chihiro, $1 \mathrm{U}$

Narita, Fumio, 1E, $1 \mathrm{Z}$

Oates, William S., 04, OT

Okubo, Kazuya, $1 \mathrm{U}$

Oppenheim, Irving J., OX

Ounaies, Zoubeida, 10

Pal, Raj K., 06

Paranjpe, Nikhil, $1 \mathrm{~K}$

Park, H. S., $1 \mathrm{~N}$

Park, J. M., $1 \mathrm{~N}$ 
Parra-Raad, Jaime, ox

Patterson, Brendan A., $1 \mathrm{~L}$

Pellegrino, Sergio, OR

Petrossian, Gayaneh, 0J

Phillips, Francis R., 08

Pöłschke, Petra, 0J, OV

Qian, Li-Jun, 1H

Rahman, Muhammad M., 1K, 24

Reich, Gregory, $1 \mathrm{~J}$

Rizvi, Reza, 18

Rosa, Matheus I. N., 06

Ruzzene, Massimo, 06

Saedi, Soheil, OH, OW

Saghaian, Ehsan, $\mathrm{OH}, \mathrm{OW}$

Salazar, Eduardo, 11

Settle, Michael, $1 \mathrm{~J}$

Shayesteh Moghaddam, Narges, $\mathrm{OH}, \mathrm{OW}, 10$

Sheikh, Rasel, 18

Shemit, Stewart, 11

Shin, P. S., 1N

Smith, David, 18

Smith, Ralph C., OS, OT, 1D

Sodano, Henry A., OZ, 1L, 1S

Solheim, Hannah, 04

Sottos, Nancy R., 11

Stanisauskis, Eugenia, 04

Stapleton, Scott, $1 \mathrm{~T}$

Takeuchi, H., $1 \mathrm{Z}$

Umer, R., OA, $1 Y$

Viet, N. V., OA, $1 Y$

Wallmersperger, Thomas, 1C, IP

Wang, Caiping, 1W

Wang, Xiaojie, 1A, 1W, 1X

Wei, Yuchen, OR

Wheeler, Robert, 08

White, Scott R., 11

White, Timothy, $1 \mathrm{~J}$

Whittaker, Molly A., OX

$\mathrm{XU}$, Xingxing, $1 \mathrm{~A}$

Yang, Zhenjun, 1E

Yoo, Jin-Hyeong, 1G

Yoshihara, Ryota, $1 \mathrm{U}$

Young, Yin LU, $1 M$

Zaki, W., OA, $1 Y$

Zhang, Li, 1D

Zhang, Wei, 10

Zhu, Honglang, IW 


\title{
Conference Committee
}

\author{
Symposium Chairs
}

Tribikram Kundu, The University of Arizona (United States)

Gregory W. Reich, Air Force Research Laboratory (United States)

Symposium Co-Chairs

Zoubeida Ounaies, The Pennsylvania State University (United States)

Hoon Sohn, KAIST (Korea, Republic of)

Conference Chair

Hani E. Naguib, University of Toronto (Canada)

Conference Co-chair

Nakhiah C. Goulbourne, University of Michigan (United States)

Conference Program Committee

Gregory P. Carman, University of California, Los Angeles (United States)

Constantin Ciocanel, Northern Arizona University (United States)

Marcelo J. Dapino, The Ohio State University (United States)

Darren J. Hartl, Texas A\&M University (United States)

Daniel J. Inman, University of Michigan (United States)

Kwang Jin Kim, University of Nevada, Las Vegas (United States)

Dimitris C. Lagoudas, Texas A\&M University (United States)

Hyeong Jae Lee, Jet Propulsion Laboratory (United States)

Donald J. Leo, Virginia Polytechnic Institute and State University (United States)

Jiangyu Li, University of Washington (United States)

Christopher S. Lynch, University of California, Los Angeles (United States)

William S. Oates, Florida State University (United States)

Zoubeida Ounaies, The Pennsylvania State University (United States)

Ralph C. Smith, North Carolina State University (United States)

Vishnu Baba Sundaresan, The Ohio State University (United States)

Session Chairs

1 Auxetic and Metamaterials

Hani E. Naguib, University of Toronto (Canada) 
Shape Memory Materials I

Oliver Myers, Clemson University (United States)

Hani E. Naguib, University of Toronto (Canada)

3 Additive Manufacturing I

Norman M. Wereley, University of Maryland, College Park (United States)

Marcelo J. Dapino, Ohio State Univeristy (United States)

Mohammad Elahinia, The University of Toledo (United States)

4 Origami Materials

Zoubeida Ounaies, The Pennsylvania State University (United States)

Paris von Lockette, The Pennsylvania State University (United States)

5 Dielectrics and Piezolectrics

Vishnu Baba Sundaresan, The Ohio State University (United States)

Amir Ameli, Washington State University Tri-Cities (United States)

6 Additive Manufacturing II

Marcelo J. Dapino, The Ohio State University (United States)

Mohammad Elahinia, The University of Toledo (United States)

7 Shape Memory Materials II

Darren J. Hartl, Texas A\&M University (United States)

Dimitris C. Lagoudas, Texas A\&M University (United States)

8 Nanocomposites Applications

Reza Rizvi, The University of Toledo (United States)

Alireza Nafari, University of Michigan (United States)

Hani E. Naguib, University of Toronto (Canada)

9 Magnetostrictive Materials

Christopher S. Lynch, University of California, Los Angeles

(United States)

Saad Ahmed, Intel Corporation (United States)

10 Mechanics of Multifunctional Materials

William S. Oates, Florida State University (United States)

11 Physically Responsive Materials

Venkata Siva Chillara, The Ohio State University (United States)

Reza Rizvi, The University of Toledo (United States)

12 Multifunctional Materials Applications

Amahadreza Jahadakbar, University of Toledo (United States)

Simone Cinquemani, Politecnico di Milano (Italy)

Alireza Nafari, University of Michigan (United States) 